\title{
Pterygium and Dry Eye- A Clinical Correlation
}

\author{
Authors \\ Dr Ann Tresa Antony, Dr Mini P.A, Dr Dalia.S \\ Department of Ophthalmology, Govt.T.D. Medical College, Alappuzha, Kerala, India \\ Corresponding Author \\ Dr Ann Tresa Antony \\ Puthussery House, Piraroor P.O, Kalady, Ernakulam, 683574 \\ Email: anntresaantony@gmail.com
}

\begin{abstract}
Purpose: To find the correlation between pterygium and dry eye.

Materials \& Methods: Hundred patients with unilateral pterygium who attended ophthalmology outpatient department at a tertiary care hospital during a period of 1 and $1 / 2$ years were included in the study. Slit lamp examination for marginal tear meniscus height, Tear film break up time, Schirmer's test with anaesthesia, Schirmer's test without anaesthesia, Rose Bengal staining were performed in both eyes of the patients. Test results were compared in pterygium eye and contralateral normal eye of the same patient. Data was entered in Microsoft Excel and SPSS 17 was used for statistical analysis.

Results: Out of the 100 patients 59 were men, while 41 were women. The median age was 51.5 years. The mean of tear film break up time, Schirmer 1, Schirmer 2, staining score were 7.6 seconds, $12.4 \mathrm{~mm}, 9.1 \mathrm{~mm}$ and 0.5 in pterygium eyes and 11.2 seconds, $17.0 \mathrm{~mm}, 14.6 \mathrm{~mm}$ and 0.2 in contralateral eyes respectively.There was a statistically significant difference in the dry eye tests results between the pterygium eyes and the contralateral eyes ( $p$ value < 0.05). The relationship of pterygium parameters (thickness, size) between tear film break up time and schirmer tests were evaluvated.Tear film break up time was significantly reduced in thick pterygium. Both tear breakup time and Schirmer's test results had no correlation with the horizontal size of pterygium. On comparison of pterygium eye with contralateral eye, Odd's Ratio was 10.44 showing increased risk of dry eye in pterygium.

Conclusion: There is a significant association between pterygium and dry eye. Pterygium leads to abnormal tear film and development of dry eye.

Keywords: Pterygium; dry eye; Schirmer's test; Tear film break up time test.
\end{abstract}

\section{INTRODUCTION}

Pterygium is a common external ocular disease in tropics. It is described as an encroachment of altered bulbar conjunctiva onto the cornea. Its name originates from the Greek word referring to its characteristic wing-like growth pattern. Pterygium is often prevalent in developing countries with scarce health resources and in this setting it can be a blinding disease. ${ }^{1}$ Pterygium is an aberrant wound healing process, characterized by centripetal growth of a leading edge of altered limbal epithelial cells, followed by a squamous metaplastic epithelium with goblet cell hyperplasia and an underlying stroma of activated, proliferating fibroblasts, neovascularization, inflammatory cells and extracellular matrix 
remodeling ${ }^{1}$. Pterygium can cause ocular irritation, induced astigmatism, tear film disturbances and decreased vision secondary to growth over visual axis. UV light exposure plays a major role in the development of pterygium.

There is a increased risk of dry eye symptoms in pterygium patients. While a direct mechanism is obvious, advances in our understanding of the inflammatory basis of dry eye syndrome suggest an indirect effect of inflammatory mediators in pterygium resulting in a dry eye state. Drying of inter palpebral tear film can be predisposing factor to pterygium. Unstable tear film in the interpalpebral area exposes the peripheral corneal epithelium, Bowman's membrane and the underlying corneal stroma to the destructive effect of UV light and the tissue damage thus sustained stimulates the formation of pterygium. Drying of interpalpebral tear film occurs most readily in the medial third of the interpalpebral fissure, because this part is farthest from lacrimal gland and nearest to the puncta.

Dry eye symptoms such as redness, irritation and blurred vision are common in pterygium. Dry eye syndrome is characterized by ocular irritation and visual disturbance resulting from alterations of the tear film and ocular surface. Dry eye is a multi factorial disease of the tears and ocular surface that results in ocular discomfort, visual disturbance and tear film instability with potential damage to the ocular surface. ${ }^{2}$ Pterygium is associated with tear hyperosmolarity and abnormal tear film function.

Whether tear dysfunction is a precursor to pterygium growth or pterygium causes tear dysfunction is still not clear. Research and clinical evidence, however, suggest that there is a relationship between the two. This study was therefore, undertaken to investigate the correlation between pterygium and dry eye. Precorneal tear film may be changed in pterygium eye. Eyes with unilateral pterygium were compared with the contralateral eyes to analyse the tear film abnormalities.

\section{MATERIALS AND METHODS}

The study was conducted in Ophthalmology department at a tertiary care hospital during a period of 1 and $1 / 2$ years. Hundred patients with unilateral pterygium who attended ophthalmology outpatient department were included in the study. Exclusion criteria were patients with primary and secondary Sjogrens syndrome, patients with ocular diseases other than pterygium leading to dry eye. Informed consent was obtained. After taking a detailed history each patient underwent a standard ophthalmological examination to exclude patients with ocular or extra ocular diseases other than pterygium that could affect tear film function such as blepharitis, ocular allergy, thyroid diseases, lacrimal system disorder, collagen diseases and use of any topical or systemic drug during the 3 month period before the examination. Age, sex, occupation, duration of growth of pterygium, history of exposure to sunlight were noted.

All patients with unilateral perygium underwent visual acuity assessment, a detailed slit-lamp examination and ophthalmosocopy to rule out adnexal, anterior segment and posterior segment diseases. The following examinations of the pterygium patients were conducted and recorded. The presence of fibro vascular tissue extending from the bulbar conjunctiva onto the cornea either nasally or temporally was accepted as pterygium. The breadth of the pterygium at the corneal periphery in millimetres and encroachment into the cornea in millimetres was described (measured by slit lamp biomicroscopy). The size of pterygium was measured from corneal limbus to apex of pterygium on a horizontal basis.

Grading of the pterygium was done according to Tan et al slit-lamp grading based on relative translucency of the body of the pterygium. ${ }^{3}$ Tear secretion and its stability were evaluated in both eyes of patients with unilateral pterygium by performing the following tests.

Examination of inferior marginal tear meniscus height with Haag-Streit slit lamp was done in both the eyes of all patients. Tear meniscus height $<$ $0.25 \mathrm{~mm}$ suggested a dry eye condition. Tear break up time test (TBUT)- Tear film breakup was 
done in all patients to assess the status of precorneal tear film The tear film breakup time is the interval between last blink and the appearance of first randomly distributed dry spot. This was repeated several times averaged. A break up time of less than 10 seconds was taken as abnormal.

Schirmers test was done in all cases using No.41 Whatman filter paper, $5 \mathrm{~mm}$ wide and $35 \mathrm{~mm}$ long. The test was performed without an topical anaesthetic (schirmer 1) and with topical anaesthtic (schirmer 2). Schirmer 2 measured basic secretion whereas schirmer 1 measured basic and reflex secretion. A measurement of less than $10 \mathrm{~mm}$ in Schirmer 1 and less than $5 \mathrm{~mm}$ in Schirmer 2 indicated dry eye. Rose Bengal staining with $1 \%$ impregnated strip was done in all cases. Van Bijsterverd grading scale was used which evaluvates the intensity of staining based on a scale of 0-3 in three areas: nasal conjunctiva, temporal conjunctiva and cornea. Score 0-3 for each zone. $1+$ : few separated spots, $2+$ : many separated spots , $3+$ : confluent spots. Maximum possible score is 9 . Scores for each eye are added and scores of 3.5 or greater indicate dry eye. ${ }^{4}$

Baseline data were entered in Microsoft Excel sheet. Data were analyzed using SPSS. Test of significance such as $t$ test for quanitative variables and chi square test for qualitative variables were done. The Pearson correlation was used to assess the correlations of tear breakup time and Schirmer's test with pterygium size. For all statistical evaluations, a two tailed probability of value $<0.05$ was considered significant.

\section{RESULTS}

Figure 1. Percentage distribution of the sample according to N/T/NT pterygium.

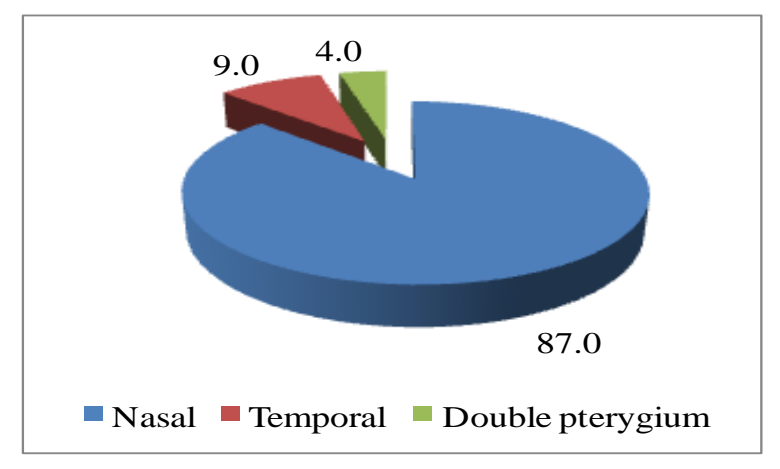

Figure 2.Comparison of TBUT mean in 2 groups.

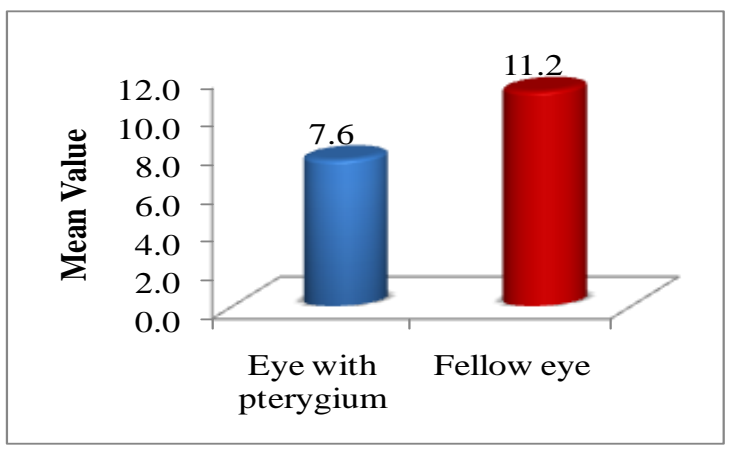

Figure 3 Comparison of Schirmer 1 mean in 2 groups.

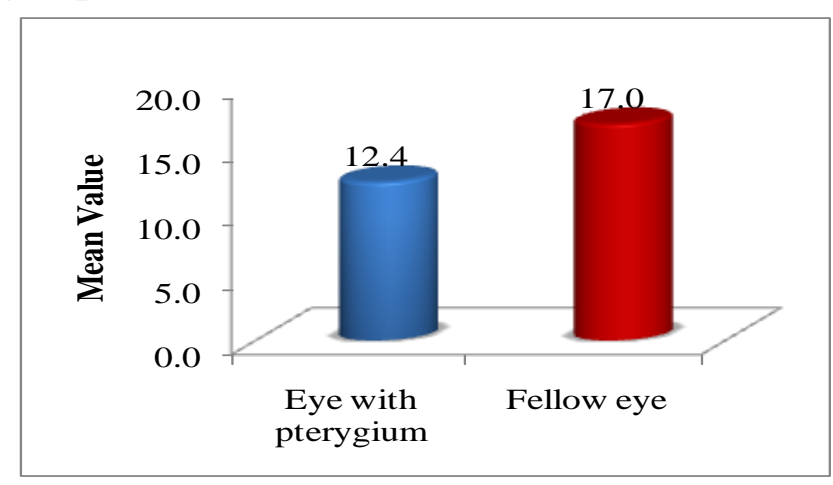

Comparison of Schirmer 2 mean in 2 groups.

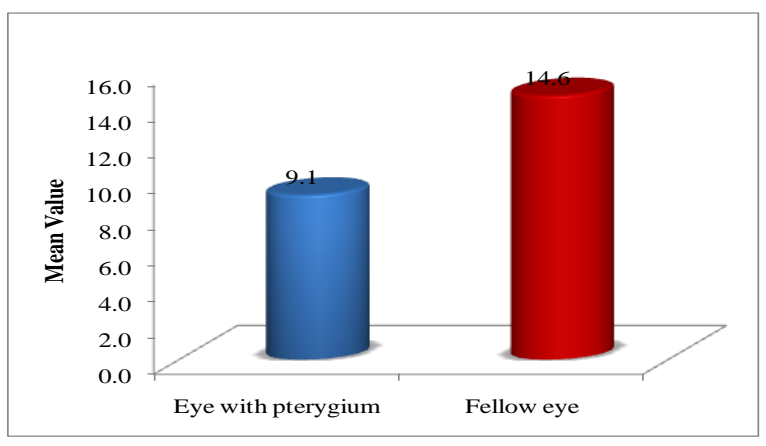

Comparison of staining score mean in 2 groups.

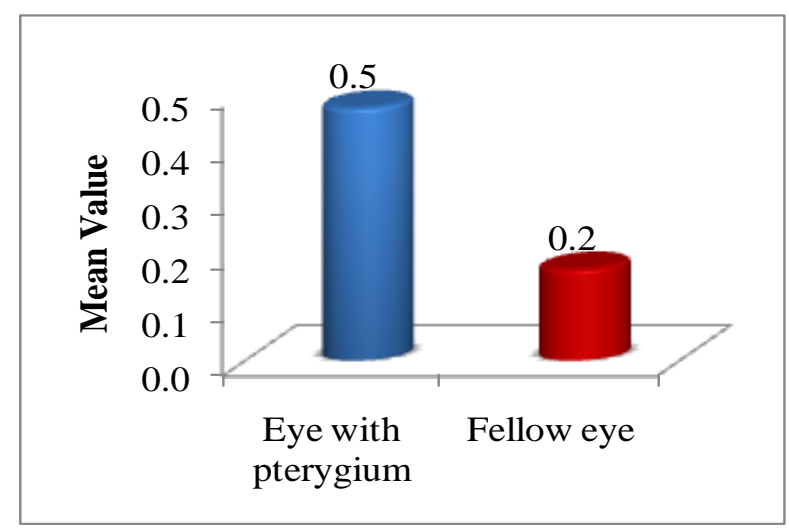




\section{DISCUSSION}

The age range of patients included in the study was 20-77 years with a mean age of 51.5 years. Out of the 100 patients studied 59 were males and 41 were females. Males spend more time in the outdoor activities and they are more exposed to damaging effects of UV rays of sunlight. A study conducted by Lee et al, 2002 also showed the prevalence of dry eye and pterygium to be more common in males. ${ }^{5}$

A strong positive correlation between climatic UV radiation and the prevalence of pterygium was found in many studies. ${ }^{6}$ Mean duration of sunlight exposure per day in our study was 3.9 hours which clearly points out UV radiation as one of the main predisposping factor leading to pterygium which ultimately lead to abnormalities in the tear film and accelerates the growth of pterygium. In our study it was found that manual labourers were the highest affected group of occupation with pterygium. Highest number of patients with pterygium was from low socioeconomic background constituting $70 \%$ of study subjects. Presence of pterygium in first degree relatives was considered as positive family history. In this study $2 \%$ patients had a family history of pterygium.

There were $61(61 \%)$ patients with pterygium in the right eye, while 39 (39\%) had it in the left. The percentage of patients affected with nasal pterygium were $87 \%$, temporalpterygium were $9 \%$, with both nasal and temporal pterygium were 4\%. Prevoius studies have shown that pterygium occur predominantly at the nasal limbus, while temporal ptergia are less common and rarely found in isolation. ${ }^{7}$ Predilection for the medial limbus is explained by chronic focal UV damage to this region which activates limbal stem cells, leading to formation of a pterygium.

Out of the 100 pateints 28 patients had Grade 1 (Tan 1), 52 patients had Grade 2 (Tan 2) and 20 patients had grade 3 (Tan 3 ) pterygium. We also measured the breadth of the pterygium at corneal limbus and mean breadth was $6.3 \mathrm{~mm}$ (SD-+ 0.8). The encroachment of pterygium onto the cornea was measured from limbus to apex of pterygium on a horizontal axis. The mean horizontal (encroachment) size of pterygium $+/$ - standard deviation was $2.1+/-0.1$ millimeters.

Marginal tear meniscus height measurement by slit lamp examination was done in pterygium eye and the fellow eye. Decreased marginal tear meniscus height $(<0.2 \mathrm{~mm})$ among the involved eyes was observed in 41 eyes while in the fellow eye it was decreased only in 8 eyes. On analysis using Chi-square test marginal tear meniscus height of pterygium eye and fellow eye was not statistically significant ( $\mathrm{p}$ value $=0.589$ ).

The mean $+/$ - standard deviation of tear breakup time in pterygium eyes was $7.6+/-2.6$ seconds and $11.2+/-1.8$ seconds in contralateral normal eyes $(\mathrm{t}=11.5$, $\mathrm{p}$ value $<0.001)$. A study conducted by Rajiv et al in pterygium and normal eyes showed mean tear film BUT of 10.4 secs in normal healthy eyes while in pterygium eyes it was reduced to 5.6 secs. ${ }^{8}$ Sukul et a1 found the mean value of tear film BUT to be 9.67 secs in Indian subjects. ${ }^{9}$

In our study the mean $+/$ - standard deviations of Schirmer 1 test results in pterygium eyes and the opposite normal eyes were $12.4+/-4.3$ and 17.0 $+/-4.3$ millimeters, respectively $(\mathrm{t}=7.47, \mathrm{p}<$ $0.001)$ which was statistically significant. In a study from India (Mithal et al, 1991), when Schirmer's test was done in two groups of patients, they found that the mean wetting of the filter paper was $12.6 \mathrm{~mm}$ and $5.2 \mathrm{~mm}$ in normal healthy eyes and the eyes of patients with pterygium. They concluded that both the values were found to be significantly reduced in cases of pterygium indicating the inadequacy of tear film in pterygium patients.

The mean $+/$ - standard deviations of Schirmer 2 test results in pterygium eyes and the opposite normal eyes were $9.1+/-3.4$ and $14.6+/$ 4.1millimeters, respectively $(\mathrm{t}=10.39, \mathrm{p}<0.001)$ which was statistically significant. Ishioka et al (2001) showed that Schirmer's test with anesthesia was shortened and was decreased in the eye with pterygium with marginal significance. They concluded that there is a correlation between pterygium formation and unstable tear film. 
On staining with Rose Bengal mean score in pterygium eyes was $0.5 \pm 1$ where as in normal eye it was $0.2 \pm 0.6$. Scores of 3.5 or greater indicate dry eye. ${ }^{10} \mathrm{On}$ comparing the mean of staining score in two groups was found to be statistically significant. Although there are so many tests available for detection and diagnosis of dry eye, the Rose Bengal test is reserved for use in severe dry eye patients with corneal signs of dry eye.

We also evaluvated the relationship of pterygium parameters ( thickness, size) between tear film breakup time and schirmer tests. Tear break up time of eye with pterygium was correlated with thickness of pterygium (based on the slit lamp grading of pterygium). On analysis we have found that in patients with grade 1 ( T 1) $67.9 \%$, Grade 2 (T 2 )78.8\%, Grade 3 (T 3 ) 100.0\% had abnormal tear film break up time. This indicates tear film break up time is reduced in thick pterygium which is found to be statistically significant ( $\mathrm{p}$ value=0.022). Schirmer 1 and Schirmer 2 tests on comparing with different grades of pterygium didn't show any statistically significant difference.

Both tear breakup time and Schirmer's test results had no correlation with pterygium size. A study by Kampitak et al also showed similar results. ${ }^{11}$ The size of pterygium was measured from limbus to apex of pterygium on a horizontal basis. The mean horizontal size of pterygium in our study was $2.1+/-0.1$ millimeters. The results of the correlation coefficient between the size of pterygium and tear breakup time were $-0.11(\mathrm{p}=$ 0.261). The correlation coefficient between pterygium size and Schirmer's test result were $0.06(p=0.52), 0.11(p=0.24)$, respectively which was not statistically significant. The odds ratio between pterygium and dry eye was 10.44. According to this study, there is a increased risk of having dry eye in pterygium eyes. Dry eye was present in $81 \%$ of eyes with pterygium and in 29 $\%$ of eyes without pterygium.

\section{CONCLUSION}

There was a statistically significant difference in the dry eye tests results between the pterygium eyes and the contralateral eyes. ( $p$ value < 0.05).On comparison of pterygium eye with contralateral eye, Odd's Ratio was 10.4 showing increased risk of dry eye in pterygium. This points out that pterygium leads to abnormal tear film and development of dry eye. This study has demonstrated that there is a strong relationship between dry eye and pterygium. Pterygium treatment should be combined with topical lubricant eye drops as as substitute of tears. Patients with unstable tear film are more prone to the damaging effects of UV rays in the sunlight. Use of protective measures like sunglasses, caps etc can provide protection against the UV rays in the sunlight and thus can help in delaying the progress of pterygium. Therefore treatment of dry eye should also be part of pterygium management.

\section{REFERENCES}

1. Coroneo MT, Chui J ,Ocular Surface Disease: Cornea, Conjunctiva and Tear Film. $2^{\text {nd }}$ Edition ;125-144.

2. Yanoff, Duker. Ophthalmology $.4^{\text {th }}$ edition ,chapter 4.23,2014;274-276.

3. Tan DTH, Chee SP, Dear KBG, Lim ASM. Effect of pterygium morphology onpterygium recurrence in a controlled trial comparing conjunctival autografting with bare sclera excision. Arch Ophthalmol. 1997; 115:1235-1240.

4. Van Bijsterveld OP. Diagnostic tests in the Siccasyndrome. Arch Ophthalmol.1969; 82:14.

5. Lee AJ, Lee J, Saw SM, Gazzard G, Koh D, Widjaja D, Tan DT (2002). Prevalence and risk factors associated with dry eye symptoms: a population based study in Indonesia. Br J Ophthalmol; 86:1347-5.

6. Panchapakesan J, Haurihan F, Mitchell P. Prevalence of pterygium and pingencula: the Blue Mountain Eye Study, Aust NZJ Ophthalmol1998; 26(1): 2-5. 
7. Dolezalova V. Is the occurrence of a temporal pterygium really so rare? Ophthalmologica 1977;174(2):88-91. Epub 1977/01/01.

8. Rajiv, Mithal S, Sood AK .Pterygium and dry eye - a clinical correlation. Indian J Ophthalmol. 2001; 39:15-6.

9. SukulR.R., ShuklaM, NagpalG.Ind. J. Ophthalmol.1983; 31-335.

10. Pflugfelder SC, Tseng SC, Yoshino K, Monroy D, Felix C, Reis BL. Correlation of goblet cell density and mucosal epithelial membrane mucin expression with rose bengal staining in patients with ocular irritation. Ophthalmology. 1997;104:223-235.

11. Kampitak K, Leelawongtawun W .Med J Assoc Thai.2014;97(5):536-539. 